RIKEN-AF-NP-214

\title{
Statistical properties of antisymmetrized molecular dynamics for non-nucleon-emission and nucleon-emission processes
}

\author{
Akira Ono \\ Institute of Physical and Chemical Research (RIKEN), Wako, Saitama 351-01, Japan \\ Hisashi Horiuchi \\ Department of physics, Kyoto University, Kyoto 606-01, Japan
}

\begin{abstract}
Statistical properties of the antisymmetrized molecular dynamics (AMD) are classical in the case of nucleon-emission processes, while they are quantum mechanical for the processes without nucleon emission. In order to understand this situation, we first clarify that there coexist mutually opposite two statistics in the AMD framework: One is the classical statistics of the motion of wave packet centroids and the other is the quantum statistics of the motion of wave packets which is described by the AMD wave function. We prove the classical statistics of wave packet centroids by using the framework of the microcanonical ensemble of the nuclear system with realistic effective twonucleon interaction. We show that the relation between the classical statistics of wave packet centroids and the quantum statistics of wave packets can be obtained by taking into account the effects of the wave packet spread. This relation clarifies how the quantum statistics of wave packets emerges from the classical statistics of wave packet centroids. It is emphasized that the temperature of the classical statistics of wave packet centroids is different from the temperature of the quantum statistics of wave packets. We then explain that the statistical properties of AMD for nucleon-emission processes are classical because nucleon-emission processes in AMD are described by the motion of wave packet centroids. We further show that when we improve the description of the nucleon-emission process so as to take into account the momentum fluctuation due to the wave packet spread, the AMD statistical properties for nucleon-emission processes change drastically into quantum statistics. Our study of nucleon-emission processes can be conversely regarded as giving another kind of proof of the fact that the statistics of wave packets is quantum mechanical while that of wave packet centroids is classical.
\end{abstract}

Typeset using REVTEX 


\section{INTRODUCTION}

In order to have better description of fragmentation processes in nuclear reactions, several molecular dynamics models with nucleon wave packets have been developed in nuclear physics besides the molecular dynamics with point nucleons imported from other fields such as molecular physics and solid state physics. An important question about the wave packet molecular dynamics is whether its statistical properties can be quantum mechanical unlike the point particle molecular dynamics or not. To have proper quantum statistics is highly desirable for the molecular dynamics description of particle emission and fragmentation processes in relatively low temperature stage of nuclear reactions.

The purpose of this paper is to clarify in detail the statistical properties of the antisymmetrized molecular dynamics (AMD) [1,2] in general situations including both non-nucleonemission and nucleon-emission processes. Many of our arguments here can be equally applied to other kinds of wave packet molecular dynamics. About the statistical properties of AMD, there have been proposed two opposite opinions, one proposed by Ohnishi and Randrup [3] who say that the AMD statistics is classical and the other proposed Schnack and Feldmeier [4] who insist that the AMD statistics is quantum mechanical as well as the statistics of the fermionic molecular dynamics (FMD) [5]. Both two groups of authors analysed the manyfermion system under harmonic oscillator mean field when they deduce their conclusions. Under this controversial situation about AMD statistics, the present authors have written recently a short paper [6] in which they have insisted that the AMD statistics is quantum mechanical for non-nucleon-emission processes while it is classical for nucleon-emission processes. In the paper the present authors have also shown that an improved treatment of nucleon-emission processes which takes into account the momentum fluctuation due to the wave packet spread converts drastically the AMD statistics for nucleon-emission processes from classical statistics into quantum statistics.

Our present paper aims to give clear arguments which resolve the above-mentioned controversial situation between Ref. [3] and Ref. [1] and then based on this clarification it aims to make the meaning of our previous paper of Ref. [6] more understandable and the arguments of it more convincing for the readers.

One of the basic points of this paper is to elucidate a unique feature of AMD that two opposite statistics are coexistent: One is the classical statistics which the centroids of nucleon wave packets obey and the other is the quantum statistics which nucleon wave packets obey in their motion described by the AMD wave function. A key point to understand this coexistent feature of different statistics is to recognize that the temperature of the classical statistics of wave packet centroids is different from the temperature which characterizes the quantum statistics of wave packets. The fact that the motion of wave packet centroids obeys classical statistics was proved by Ohnishi and Randrup [3] by using the canonical ensemble technique in simple cases of many-fermion system under harmonic oscillator mean field and also in free gas state. In this paper, we prove this fact by using the microcanonical ensemble technique in the case of realistic many-nucleon system interacting with effective two-nucleon force. The fact that the motion of wave packets described by the AMD wave function obeys the quantum statistics was proved by Schnack and Feldmeier [四] also in the case of harmonic oscillator many-fermion system. Their proof was made by calculating the occupation probability of the harmonic oscillator single-particle level by performing the 
long-time average in AMD (or FMD) and then by showing that the occupation probability calculated with AMD (or FMD) has just the same value as that given by the quantum mechanical canonical ensemble average. In this paper we prove this fact in another way so that we can understand the relation between the classical statistics of wave packet centroids and the quantum statistics of wave packets. Namely we will show that, when we calculate the occupation probability of the harmonic oscillator single-particle level on the basis of the fact that wave packet centroids obey classical statistics, the resulting value of the occupation probability proves to be almost the same as the value given by the quantum mechanical canonical ensemble average. Through this proof we will see that the transition from the classical statistics of wave packet centroids to the quantum statistics of wave packets is surely due to effects of the wave packet spread.

In the case of the harmonic oscillator many-fermion system which both Ohnishi-Randrup and Schnack-Feldmeier groups treated, the AMD statistics should be regarded as being quantum mechanical just in accordance with the opinion of Schnack and Feldmeier. It is because the AMD statistics that is relevant for calculated observables is not the statistics of wave packet centroids but that of wave packets whose motion is described by the AMD wave function. We should note that the calculation of observables is made by the use of the AMD wave function. We can say that the AMD statistics in the case of the realistic nuclear system is also quantum mechanical so long as we are concerned with dynamical processes confined spatially inside the nucleus like the harmonic oscillator many-fermion system.

However, as we discussed in our previous paper [6], the situation changes when we treat nucleon-emission processes with AMD. The nucleon-emission process can not be studied with the harmonic oscillator many-fermion model nor the free gas model. The AMD statistics which is contained in nucleon-emission processes is now not the statistics of wave packets but the statistics of the wave packet centroids and hence it is classical. The reason is because the nucleon emission process in AMD is governed by the motion of the wave packet centroid. In AMD, a nucleon cannot go out from a nucleus if the wave packet centroid cannot go out from the nucleus even when the high momentum component of the wave packet can go out from the nucleus if treated quantum mechanically. In Ref. [6] we further showed that we can recover the quantum mechanical character of the AMD statistics also for nucleon-emission processes by introducing a modified treatment of the nucleon-emission process. An essential point of the modification is that we allow a wave packet near the nuclear surface to split into high and low momentum components and allow the high momentum component to escape from the nucleus. Namely we take into account dynamical effects of the momentum fluctuation due to the wave packet spread. In this paper we give a detailed discussion of this modification on the basis of our clarified recognition of the coexistence of mutually opposite two statistics in the AMD framework. Our study of nucleon-emission processes with the ordinary AMD and with the modified AMD can be conversely used as another kind of proof of the fact that the statistics of wave packets is quantum mechanical while that of wave packet centroids is classical.

The organization of this paper is as follows. In the next section (Sec. II) we explain briefly the AMD formalism. Then in Sec. III, we prove that the statistical properties of wave packet centroids are classical. The proof is performed by using the microcanonical ensemble technique in the case of realistic many-nucleon system interacting with effective two-nucleon force. In Sec. IV we prove that the statistical properties of wave packets whose 
motion is described by the AMD wave function are quantum mechanical. The proof is made in the case of the harmonic oscillator many-fermion system by calculating numerically the occupation probability of the harmonic oscillator single-particle level on the basis of the fact that wave packet centroids obey the classical statistics. We will see that the resulting value of the occupation probability proves to be almost the same as the value given by the quantum mechanical canonical ensemble average. Here we further show that in the case of the harmonic oscillator system of distinguishable many particles the proof can be made totally analytically. In Sec. V we discuss the statistical properties of AMD for nucleonemission processes. We then discuss a modified treatment of the nucleon-emission process which recovers the quantum mechanical character of the AMD statistics also for the nucleonemission process. Summarizing discussions are given in Sec. VI.

\section{BRIEF EXPLANATION OF AMD FORMALISM}

We here explain briefly the AMD formalism. For details the reader is referred to Ref. [1]. AMD describes the nuclear many-body system by a Slater determinant of Gaussian wave packets as

$$
\begin{aligned}
\Phi(Z) & =\operatorname{det}\left[\phi\left(\mathbf{r}_{j}, \mathbf{Z}_{k}\right) \chi_{\alpha_{k}}(j)\right] \\
\phi(\mathbf{r}, \mathbf{Z}) & =\left(\frac{2 \nu}{\pi}\right)^{3 / 4} \exp \left\{-\nu(\mathbf{r}-\mathbf{Z} / \sqrt{\nu})^{2}+\frac{1}{2} \mathbf{Z}^{2}\right\}
\end{aligned}
$$

where the complex parameters $Z \equiv\left\{\mathbf{Z}_{k}\right\}$ stand for the centroids of wave packets and $\chi_{\alpha}(j)$ for the spin-isospin wave function with $\alpha=\mathrm{p} \uparrow, \mathrm{p} \downarrow, \mathrm{n} \uparrow, \mathrm{n} \downarrow$. The width parameter $\nu$ is common to all nucleons and is time-independent. The time evolution of $Z$ is determined by the time-dependent variational principle and the stochastic two-nucleon collision process. The AMD equation of motion for $Z$ derived from the time-dependent variational principle is

$$
\begin{aligned}
& i \hbar \sum_{k \tau} C_{j \sigma, k \tau} \frac{d Z_{k \tau}}{d t}=\frac{\partial \mathcal{H}}{\partial Z_{j \sigma}^{*}}, \\
& C_{j \sigma, k \tau}=\frac{\partial^{2}}{\partial Z_{j \sigma}^{*} \partial Z_{k \tau}} \log \langle\Phi(Z) \mid \Phi(Z)\rangle,
\end{aligned}
$$

where $\sigma, \tau=x, y, z$ and the Hamiltonian function $\mathcal{H}$ is the expectation value of the Hamiltonian operator $H$ with the subtraction of the spurious kinetic energies of the center-of-mass zero-point oscillations of fragments,

$$
\mathcal{H}=\frac{\langle\Phi(Z)|H| \Phi(Z)\rangle}{\langle\Phi(Z) \mid \Phi(Z)\rangle}-\frac{3 \hbar^{2} \nu}{2 M} A+T_{0}\left(A-N_{F}\right) .
$$

Here $A$ is the total mass number, $M$ is the nucleon mass, $N_{F}$ is the fragment number which is a function of $Z$ and $Z^{*}$, and $T_{0}$ is $3 \hbar^{2} \nu / 2 M$ in principle but is treated usually as a free parameter for the adjustment of binding energies.

For the stochastic two-nucleon process, we introduce physical nucleon coordinates $W=$ $\left\{\mathbf{W}_{j}\right\}$ as 


$$
\begin{aligned}
\mathbf{W}_{j} & =\sum_{k=1}^{A}(\sqrt{Q})_{j k} \mathbf{Z}_{k}, \\
Q_{j k} & =\frac{\partial}{\partial\left(\mathbf{Z}_{j}^{*} \cdot \mathbf{Z}_{k}\right)} \log \langle\Phi(Z) \mid \Phi(Z)\rangle .
\end{aligned}
$$

In the case of light systems where we have less than three nucleons in any spin-isospin state, the physical nucleon coordinates $W$ are canonical coordinates. However in other cases $W$ are not canonical coordinates. An important property of $W$ is that in the many-body phase space of $W$ there exists a Pauli-forbidden region into which any nucleon cannot enter. For example, we can easily show that the following region of $W$ is contained in the Pauliforbidden region,

$$
\sum_{j=1}^{A} \mathbf{W}_{j}^{*} \cdot \mathbf{W}_{j}<N_{A},
$$

where $N_{A}$ is the minimum number of the total harmonic oscillator quanta of the $A$-nucleon system. The Pauli blocking of the two-nucleon collision is defined such that the two-nucleon collision should avoid entering into the Pauli-forbidden region.

The AMD equation of motion (Eq. (21)) is not of canonical form, indicating that the coordinates $Z$ are not canonical coordinates. Although the existence of canonical coordinates is known mathematically, we do not know at present the explicit forms of canonical coordinates in AMD except in the case of light systems mentioned above (just below Eq. (4⿴囗十)). However, even if we do not know the explicit forms of canonical coordinates, we can derive the explicit form of the Jacobian of the transformation between canonical coordinates and the original coordinates $Z$. If we express a set of canonical coordinates by $Y=\left\{\mathbf{Y}_{j}\right\}$, the Jacobian $J$ can be shown to be given by the determinant of the hermitian matrix $\left\{C_{j \sigma, k \tau}\right\}$ appearing in the AMD equation of motion (Eq. (21)),

$$
J=\frac{\partial\left(Y, Y^{*}\right)}{\partial\left(Z, Z^{*}\right)}=\frac{\partial(R, P)}{\partial(D, K)}=\operatorname{det}\left[C_{j \sigma, k \tau}\right],
$$

where $R=\left\{\mathbf{R}_{j}\right\}$ and $P=\left\{\mathbf{P}_{j}\right\}$ are real and imaginary parts of $Y$, respectively, while $D=$ $\left\{\mathbf{D}_{j}\right\}$ and $K=\left\{\mathbf{K}_{j}\right\}$ are those of $Z$ :

$$
\mathbf{Y}_{j}=\sqrt{\nu} \mathbf{R}_{j}+\frac{i}{2 \hbar \sqrt{\nu}} \mathbf{P}_{j}, \quad \mathbf{Z}_{j}=\sqrt{\nu} \mathbf{D}_{j}+\frac{i}{2 \hbar \sqrt{\nu}} \mathbf{K}_{j} .
$$

In spite of our general ignorance about explicit forms of canonical coordinates, there exists one canonical coordinate whose explicit form we know. It is the center of mass coordinate $\mathbf{Z}_{G}$,

$$
\begin{aligned}
\mathbf{Z}_{G} & =\frac{1}{\sqrt{A}} \sum_{j=1}^{A} \mathbf{Z}_{j}=\sqrt{A \nu} \mathbf{D}_{G}+\frac{i}{2 \hbar \sqrt{A \nu}} \mathbf{K}_{G}, \\
\mathbf{D}_{G} & =\frac{1}{A} \sum_{j=1}^{A} \mathbf{D}_{j}, \quad \mathbf{K}_{G}=\sum_{j=1}^{A} \mathbf{K}_{j} .
\end{aligned}
$$


For the sake of discussions in later sections, we here emphasize the fact that the AMD wave function is just the exact solution of the time-dependent Schrödinger equation in the case of the harmonic oscillator mean field. Namely, when the Hamiltonian operator $H$ is given as

$$
H=\hbar \omega \sum_{j=1}^{A} \mathbf{a}_{j}^{\dagger} \cdot \mathbf{a}_{j}+E_{0},
$$

where $\mathbf{a}_{j}^{\dagger}$ and $\mathbf{a}_{j}(j=1 \sim A)$ are harmonic oscillator creation and annihilation operators, the exact solution of the Schrödinger equation $i \hbar(\partial / \partial t) \Psi=H \Psi$ is given as

$$
\Psi=e^{-i E_{0} t / \hbar} \operatorname{det}\left[\phi\left(\mathbf{r}_{j}, e^{-i \omega t} \mathbf{Z}_{k}^{(0)}\right) \chi_{\alpha_{k}}(j)\right] .
$$

Here the width parameter $\nu$ of the Gaussian wave packet $\phi$ is $\nu=M \omega / 2 \hbar$, and the parameters

$\left\{\mathbf{Z}_{k}^{(0)}\right\}$ stand for arbitrary initial values for $Z=\left\{\mathbf{Z}_{k}\right\}$. Needless to say, $\left\{\mathbf{Z}_{k}=e^{-i \omega t} \mathbf{Z}_{k}^{(0)},(k=\right.$ $1 \sim A)\}$ are the solution of the AMD equation of motion (Eq. (2)) with $T_{0}=3 \hbar \nu / 2 M$. We should note the fact that the AMD wave function which is the exact solution of the Schrödinger equation is constructed from the motion of wave packet centroids which make just the classical oscillatory motion $\left\{\mathbf{Z}_{k}=e^{-i \omega t} \mathbf{Z}_{k}^{(0)},(k=1 \sim A)\right\}$. This fact constitutes the basic background of the character of the AMD statistical properties which we clarify in later sections. There we will see that the quantum statistics of AMD is constructed from the statistics of wave packet centroids which is classical.

\section{CLASSICAL STATISTICS OF WAVE PACKET CENTROIDS}

We here prove the fact that the motion of wave packet centroids in AMD obeys classical statistics. The statistical properties of wave packet centroids are determined by the AMD equation of motion. We consider that stochastic two-nucleon collisions are not relevant to the character of the statistics but play only the role to hasten the equilibration of the system. The AMD equation of motion of Eq. (2) is not of canonical form but it can be converted into the canonical form if we use canonical coordinates instead of $Z$. Hence the dynamics of wave packet centroids is classical and the statistical properties of wave packet centroids are expected to be classical. However, what inhibits us from concluding straightforwardly in this way is the effect of the antisymmetrization contained in the AMD equation of motion. As we explained in Sec. II, there exists a Pauli-forbidden region in the phase space of the physical coordinates $W$. Although the physical coordinates $W$ are not always equivalent to canonical coordinates, we can guess the existence of a Pauli-forbidden region also in the phase space of canonical coordinates. If we have a Pauli-forbidden region, we are not sure whether we can simply say or not that the statistics of wave packet centroids is classical. Under this situation, we are forced to check explicitly the character of the statistics of wave packet centroids.

In Ref. [3], Ohnishi and Randrup investigated the character of the statistics of wave packet centroids by using the canonical ensemble technique in simple cases of many-fermion system under one-dimensional harmonic oscillator mean field and also in free gas state. The 
result they obtained is that the statistics of wave packet centroids is classical. Here in this paper, we investigate the the character of the statistics of wave packet centroids by using the microcanonical ensemble technique in the case of realistic many-nucleon system interacting with effective two-nucleon force.

In the microcanonical ensemble method, the relation between the energy $E$ and the temperature $T_{c}$ is given by

$$
\begin{gathered}
\frac{1}{T_{c}}=\frac{\partial \log \rho(E)}{\partial E} \\
\rho(E)=\int\left(\prod_{j=1}^{A} \frac{d^{3} R_{j} d^{3} P_{j}}{(2 \pi \hbar)^{3}}\right) \delta(\mathcal{H}-E) \delta\left(\mathbf{D}_{G}\right) \delta\left(\mathbf{K}_{G}\right) \\
=\int\left(\prod_{j=1}^{A} \frac{d^{3} D_{j} d^{3} K_{j}}{(2 \pi \hbar)^{3}}\right) \operatorname{det}[C] \delta(\mathcal{H}-E) \delta\left(\mathbf{D}_{G}\right) \delta\left(\mathbf{K}_{G}\right) .
\end{gathered}
$$

Here $\operatorname{det}[C]=\operatorname{det}\left[C_{j \sigma, k \tau}\right]$ and we have used the relation of Eq. (6) for the Jacobian between $Z$ and the canonical coordinates $Y$. The existence of $\delta\left(\mathbf{D}_{G}\right) \delta\left(\mathbf{K}_{G}\right)$ is because of the fact that the center of mass coordinate $\mathbf{Z}_{G}(\mathrm{Eq}$. (8)) is a conserved quantity and makes no contribution to the phase volume $\rho(E)$ of the internal motion of the nucleus. For the sake of the numerical evaluation of this relation, we rewrite this relation as explained below [7]. We first notice the following identity relation

$$
\begin{aligned}
& \frac{\partial}{\partial E} \int d Z \operatorname{det}[C] \delta(\mathcal{H}-E) \delta\left(\mathbf{D}_{G}\right) \delta\left(\mathbf{K}_{G}\right) \sum_{\alpha=1}^{6 A} a_{\alpha} \frac{\partial \mathcal{H}}{\partial z_{\alpha}} \\
& =\int d Z \operatorname{det}[C] \delta(\mathcal{H}-E) \delta\left(\mathbf{D}_{G}\right) \delta\left(\mathbf{K}_{G}\right) \sum_{\alpha=1}^{6 A}\left(\frac{\partial a_{\alpha}}{\partial z_{\alpha}}+a_{\alpha} \frac{\partial \log \operatorname{det}[C]}{\partial z_{\alpha}}+a_{\alpha} \frac{\partial \log \delta\left(\mathbf{D}_{G}\right) \delta\left(\mathbf{K}_{G}\right)}{\partial z_{\alpha}}\right)
\end{aligned}
$$

where

$$
\begin{gathered}
d Z=\prod_{j=1}^{A} \frac{d^{3} D_{j} d^{3} K_{j}}{(2 \pi \hbar)^{3}}, \\
\left\{z_{\alpha}, \alpha=1 \sim 6 A\right\}=\left\{\mathbf{D}_{j}, \mathbf{K}_{j}, j=1 \sim A\right\},
\end{gathered}
$$

and $\left\{a_{\alpha}\right\}$ are arbitrary functions of $Z$. The identity relation of Eq. (12) can be easily derived by using $(\partial / \partial E \delta(\mathcal{H}-E))\left(\partial \mathcal{H} / \partial z_{\alpha}\right)=-\partial / \partial z_{\alpha} \delta(\mathcal{H}-E)$ and integration by parts. We choose $\left\{a_{\alpha}\right\}$ so that they satisfy

$$
\sum_{\alpha} a_{\alpha} \frac{\partial \mathbf{D}_{G}}{\partial z_{\alpha}}=0, \quad \sum_{\alpha} a_{\alpha} \frac{\partial \mathbf{K}_{G}}{\partial z_{\alpha}}=0 .
$$

For those $\left\{a_{\alpha}\right\}$ which satisfy Eq. (14), we have

$$
\sum_{\alpha} a_{\alpha} \frac{\partial \log \delta\left(\mathbf{D}_{G}\right) \delta\left(\mathbf{K}_{G}\right)}{\partial z_{\alpha}}=0 .
$$

By dividing both sides of the identity relation Eq. (12) by $\rho(E)$ and by using Eq. (11), we get 


$$
T_{c}=\frac{\left\langle\sum_{\alpha} a_{\alpha} \frac{\partial \mathcal{H}}{\partial z_{\alpha}}\right\rangle}{\left\langle\sum_{\alpha}\left(\frac{\partial a_{\alpha}}{\partial z_{\alpha}}+a_{\alpha} \frac{\partial \log \operatorname{det}[C]}{\partial z_{\alpha}}\right)\right\rangle-\frac{\partial}{\partial E}\left\langle\sum_{\alpha} a_{\alpha} \frac{\partial \mathcal{H}}{\partial z_{\alpha}}\right\rangle},
$$

where $\langle Q(Z)\rangle$ stands for the microcanonical ensemble average of the quantity $Q(Z)$ for the energy $E$ and is defined as

$$
\langle Q(Z)\rangle=\frac{\int d Z \operatorname{det}[C] \delta(\mathcal{H}-E) \delta\left(\mathbf{D}_{G}\right) \delta\left(\mathbf{K}_{G}\right) Q(Z)}{\rho(E)} .
$$

Under the thermally equilibrated situation of the system, we can equate the microcanonical ensemble average $\langle Q(Z)\rangle$ to the long-time average $\overline{Q(Z)}$;

$$
\begin{aligned}
\langle Q(Z)\rangle & =\overline{Q(Z)} \\
\overline{Q(Z)} & =\lim _{t_{2} \rightarrow \infty} \frac{1}{t_{2}-t_{1}} \int_{t_{1}}^{t_{2}} d t Q(Z(t)) .
\end{aligned}
$$

By using this equivalence of $\langle Q(Z)\rangle$ with $\overline{Q(Z)}$, we can numerically evaluate Eq. (16) by calculating the time development of $Z$ with the AMD equation of motion for a long time. We give in Fig. 1 the calculated relation between the temperature $T_{c}$ and the energy $E$ in the system of ${ }^{12} \mathrm{C}$. Since the contribution of the term $(\partial / \partial E)\left\langle\sum_{\alpha} a_{\alpha} \partial \mathcal{H} / \partial z_{\alpha}\right\rangle$ in the denominator of Eq. (16) is very small, we neglected this term in the calculation. The arbitrary functions $\left\{a_{\alpha}\right\}$ were here selected to be

$$
\begin{array}{ll}
a_{\alpha}=z_{\alpha}-\frac{1}{A} \sum_{k=1}^{A} D_{k \sigma} \quad \text { for } \quad z_{\alpha}=D_{j \sigma}, \\
a_{\alpha}=z_{\alpha}-\frac{1}{A} \sum_{k=1}^{A} K_{k \sigma} \quad \text { for } \quad z_{\alpha}=K_{j \sigma} .
\end{array}
$$

We can easily check that these $\left\{a_{\alpha}\right\}$ surely satisfy Eq. (14). We adopted the Volkov No.1 force [8] for the effective two-nucleon force. The oscillator width $\nu$ was chosen to be $\nu=0.16 \mathrm{fm}^{-2}$. It is easy to see that the relation between the excitation energy $E^{*}=E-E$ (ground state) and the temperature $T_{c}$ is very close to the caloric curve $E^{*} / 11=3 T_{c}$. Since this means that the specific heat remains constant even around zero temperature, the statistics is not quantum mechanical. It is well known that the caloric curve $E^{*} / A=3 T_{c}$ is characteristic to the classical statistics of the harmonic oscillator many-body system. We can say that the reason why the caloric curve in Fig. 1 is closer to $E^{*} / 11=3 T_{c}$ than $E^{*} / 12=3 T_{c}$ is because in our present approach by the use of the microcanonical ensemble, the center of mass coordinate is not involved in the thermal motion implying that the number of degrees of freedom is $3(A-1)$ rather than $3 A$. We thus conclude that the statistics of AMD wave packet centroids for the realistic nuclear system is classical. 


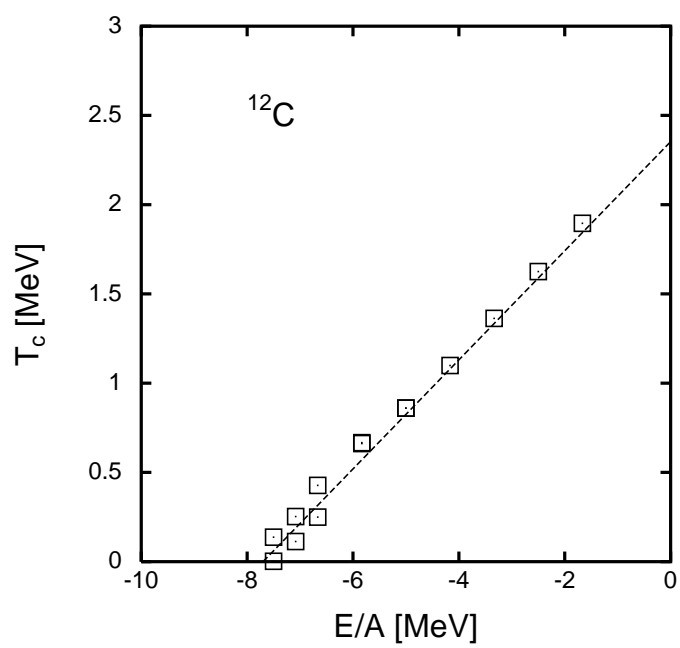

FIG. 1. The relation between the temperature $T_{c}$ and the energy $E$ for the system of ${ }^{12} \mathrm{C}$. Squares are the calculated results with the microcanonical ensemble method. Different values of $T_{c}$ for the same $E$ is due to the statistical error. Dotted line shows the relation $E^{*} /(A-1)=3 T_{c}$ with $A=12$.

\section{QUANTUM STATISTICS OF WAVE PACKETS}

The fact that the motion of wave packets described by the AMD wave function obeys the quantum statistics was first given an explicit proof by Schnack and Feldmeier [4]. They treated the same model system as Ohnishi and Randrup [3], namely the one-dimensional harmonic oscillator many-fermion system. They also treated the harmonic oscillator system of distinguishable particles and found also the quantum mechanical character for the statistics of wave packets. Their proof was made by calculating the occupation probability of the harmonic oscillator single-particle level by performing the long-time average in AMD (or FMD) and then by showing that the occupation probability calculated with AMD (or FMD) has just the same value as that given by the quantum mechanical canonical ensemble average. Here in this paper, we prove the quantum statistics of wave packets in another way so that we can understand its relation with the classical statistics of wave packet centroids. Our proof is made by treating the same one-dimensional harmonic oscillator systems of fermions and also of distinguishable particles.

Like Schnack and Feldmeier, we investigate the occupation probability of the harmonic oscillator single-particle level. In AMD, this quantity which we express as $\mathcal{P}_{n}(Z)$ for the AMD wave function $\Phi(Z)$ is given by

$$
\begin{aligned}
& \mathcal{P}_{n}(Z)=\frac{\left\langle\Phi(Z)\left|P_{n}\right| \Phi(Z)\right\rangle}{\langle\Phi(Z) \mid \Phi(Z)\rangle}, \\
& P_{n}=C_{n}^{\dagger} C_{n}=\sum_{j=1}^{A}\left|\phi_{n}\left(x_{j}\right)\right\rangle\left\langle\phi_{n}\left(x_{j}\right)\right|,
\end{aligned}
$$

where $\phi_{n}(x)$ is the one-dimensional harmonic oscillator eigenfuncion with oscillator quanta $n$. Here the AMD wave function $\Phi(Z)$ should be considered to be a Slater determinant of one-dimensional Gaussian wave packet $\phi\left(x, Z_{j}\right)=\sqrt{2 \nu / \pi} \exp \left\{-\nu\left(x-Z_{j} / \sqrt{\nu}\right)^{2}+(1 / 2) Z_{j}^{2}\right\}$ without spin-isospin wave function (or belonging to a single spin-isospin state). The investigation of $\mathcal{P}_{n}(Z)$ is made in thermally equilibrated situation of the system. In this section we 
describe the thermal equilibrium by the canonical ensemble method. It means that we investigate the statistical ensemble of AMD wave functions whose centroids $Z$ follow the classical canonical statistics. Let $T_{c}$ denote the temperature of the motion of wave packet centroids. The average value of $\mathcal{P}_{n}(Z)$ under thermal equilibrium, which we express as $\left\langle\mathcal{P}_{n}(Z)\right\rangle_{T_{c}}$, is calculated as

$$
\begin{aligned}
\left\langle\mathcal{P}_{n}(Z)\right\rangle_{T_{c}} & =\frac{\int d Z \operatorname{det}[C] \mathcal{P}_{n}(Z) e^{-\mathcal{H}(Z) / T_{c}}}{\int d Z \operatorname{det}[C] e^{-\mathcal{H}(Z) / T_{c}}}, \\
\mathcal{H}(Z) & =\frac{\left\langle\Phi(Z)\left|\hbar \omega \sum_{j=1}^{A}\left(a_{j}^{\dagger} a_{j}+1 / 2\right)\right| \Phi(Z)\right\rangle}{\langle\Phi(Z) \mid \Phi(Z)\rangle} .
\end{aligned}
$$

Here the notations, $d Z, \operatorname{det}[C]$, and others are obvious one-dimensional analogues of the corresponding three-dimensional quantities; for example,

$$
\begin{aligned}
d Z & =\prod_{j=1}^{A} \frac{d D_{j} d K_{j}}{2 \pi \hbar} \\
\operatorname{det}[C] & =\operatorname{det}\left[\frac{\partial^{2}}{\partial Z_{j}^{*} \partial Z_{k}} \log \langle\Phi(Z) \mid \Phi(Z)\rangle\right] .
\end{aligned}
$$

Similarly, the average value of the energy under thermal equilibrium, which we express as $\langle\mathcal{H}(Z)\rangle_{T_{c}}$, is calculated as

$$
\langle\mathcal{H}(Z)\rangle_{T_{c}}=\frac{\int d Z \operatorname{det}[C] \mathcal{H}(Z) e^{-\mathcal{H}(Z) / T_{c}}}{\int d Z \operatorname{det}[C] e^{-\mathcal{H}(Z) / T_{c}}} .
$$

We have numerically calculated $\left\langle\mathcal{P}_{n}(Z)\right\rangle_{T_{c}}$ and $\langle\mathcal{H}(Z)\rangle_{T_{c}}$ by using the Metropolis sampling method as in Ref. [3] in the case of the system with fermion number $4(A=4)$. The quantity $\langle\mathcal{H}(Z)\rangle_{T_{c}}$ is just the quantity which Ohnishi and Randrup calculated in Ref. [3]. We have ascertained their result in the case of $A=4$; namely the calculated value of $\langle\mathcal{H}(Z)\rangle_{T_{c}}$ is given almost exactly as

$$
\langle\mathcal{H}(Z)\rangle_{T_{c}}=A T_{c}+\hbar \omega \sum_{n=0}^{A-1}\left(n+\frac{1}{2}\right) .
$$

We suspect that this relation is analytically exact, since our numerical calculation shows that this equality holds with very high accuracy. This result, of course, shows that the motion of wave packet centroids obeys the classical statistics.

Now let us consider the quantity $\left\langle\mathcal{P}_{n}(Z)\right\rangle_{T_{c}}$. In classical mechanics, we have no such quantity as $\left\langle\mathcal{P}_{n}(Z)\right\rangle_{T_{c}}$, because there is no concept of the discrete energy level in classical mechanics. Therefore we have no idea what value the quantity $\left\langle\mathcal{P}_{n}(Z)\right\rangle_{T_{c}}$ should take in the case of classical statistics. One may think that we have the relation $\left\langle\mathcal{P}_{n}(Z)\right\rangle_{T_{c}} \propto \exp \left(-n \hbar \omega / T_{c}\right)$ with some small modification by the Pauli principle. However, we will soon see that the $n$ dependence of the calculated values of $\left\langle\mathcal{P}_{n}(Z)\right\rangle_{T_{c}}$ is very different from that of $\exp \left(-n \hbar \omega / T_{c}\right)$. 
Since the quantity $\left\langle\mathcal{P}_{n}(Z)\right\rangle_{T_{c}}$ is a quantum mechanical quantity, we should check whether the calculated values of $\left\langle\mathcal{P}_{n}(Z)\right\rangle_{T_{c}}$ are equal or not to what the quantum statistics gives us.

In the quantum statistics, the average value of the occupation probability of the harmonic oscillator single-particle level, which we express as $\left\langle\left\langle P_{n}\right\rangle\right\rangle_{T}$, is given as

$$
\begin{aligned}
\left\langle\left\langle P_{n}\right\rangle\right\rangle_{T} & =\frac{\sum_{n_{1} \ldots n_{4}}{ }^{a}\left\langle n_{1} \ldots n_{4}\left|P_{n}\right| n_{1} \ldots n_{4}\right\rangle^{a} e^{-E\left(n_{1} \ldots n_{4}\right) / T}}{\sum_{n_{1} \ldots n_{4}}{ }^{a}\left\langle n_{1} \ldots n_{4} \mid n_{1} \ldots n_{4}\right\rangle^{a} e^{-E\left(n_{1} \ldots n_{4}\right) / T}}, \\
\left|n_{1} \ldots n_{4}\right\rangle^{a} & =\frac{1}{\sqrt{4 !}} \operatorname{det}\left[\phi_{n_{1}}\left(x_{1}\right) \ldots \phi_{n_{4}}\left(x_{4}\right)\right], \\
E\left(n_{1} \ldots n_{4}\right) & =\sum_{j=1}^{4}\left(n_{j}+\frac{1}{2}\right) \hbar \omega .
\end{aligned}
$$

Similarly, in the quantum statistics, the average value of the energy, which we express as $\langle\langle H\rangle\rangle_{T}$, is given as

$$
\langle\langle H\rangle\rangle_{T}=\frac{\sum_{n_{1} \ldots n_{4}}{ }^{a}\left\langle n_{1} \ldots n_{4} \mid n_{1} \ldots n_{4}\right\rangle^{a} E\left(n_{1} \ldots n_{4}\right) e^{-E\left(n_{1} \ldots n_{4}\right) / T}}{\sum_{n_{1} \ldots n_{4}}{ }^{a}\left\langle n_{1} \ldots n_{4} \mid n_{1} \ldots n_{4}\right\rangle^{a} e^{-E\left(n_{1} \ldots n_{4}\right) / T}} .
$$

The necessary and sufficient condition that the system has quantum statistical properties is that any of thermally averaged quantities like $\left\langle\mathcal{P}_{n}(Z)\right\rangle_{T_{c}}$ and $\langle\mathcal{H}(Z)\rangle_{T_{c}}$ are expressed by the thermally averaged quantities in quantum canonical ensemble with a certain temperature $T$. What is decisively important here is the recognition that there is no need that the temperature $T$ of the quantum statistics is equal to the temperature $T_{c}$ of wave packet centroids. The relation between the temperatures $T$ and $T_{c}$ can be obtained for example by the requirement that the value of $\langle\mathcal{H}(Z)\rangle_{T_{c}}$ is equal to the value $\langle\langle H\rangle\rangle_{T}$.

We here summarize as follows what we are going to check now: We first calculate numerically the relation between $T$ and $T_{c}$ from the relation

$$
\langle\langle H\rangle\rangle_{T}=\langle\mathcal{H}(Z)\rangle_{T_{c}},
$$

Then we check whether the relation,

$$
\left\langle\left\langle P_{n}\right\rangle\right\rangle_{T}=\left\langle\mathcal{P}_{n}(Z)\right\rangle_{T_{c}}
$$

holds or not. In Fig. 2 we show that the numerical calculations assure very well the relation of Eq. (28). This result demonstrates clearly that the statistics of the motion of wave packets described by the AMD wave function is quantum mechanical.

Our arguments given above in this section can be made completely analytically in the case of distinguishable particles. For distinguishable particles, the AMD wave function is simply a product of Gaussian wave packets,

$$
\Phi(Z)=\prod_{j=1}^{A} \phi\left(x_{j}, Z_{j}\right) .
$$



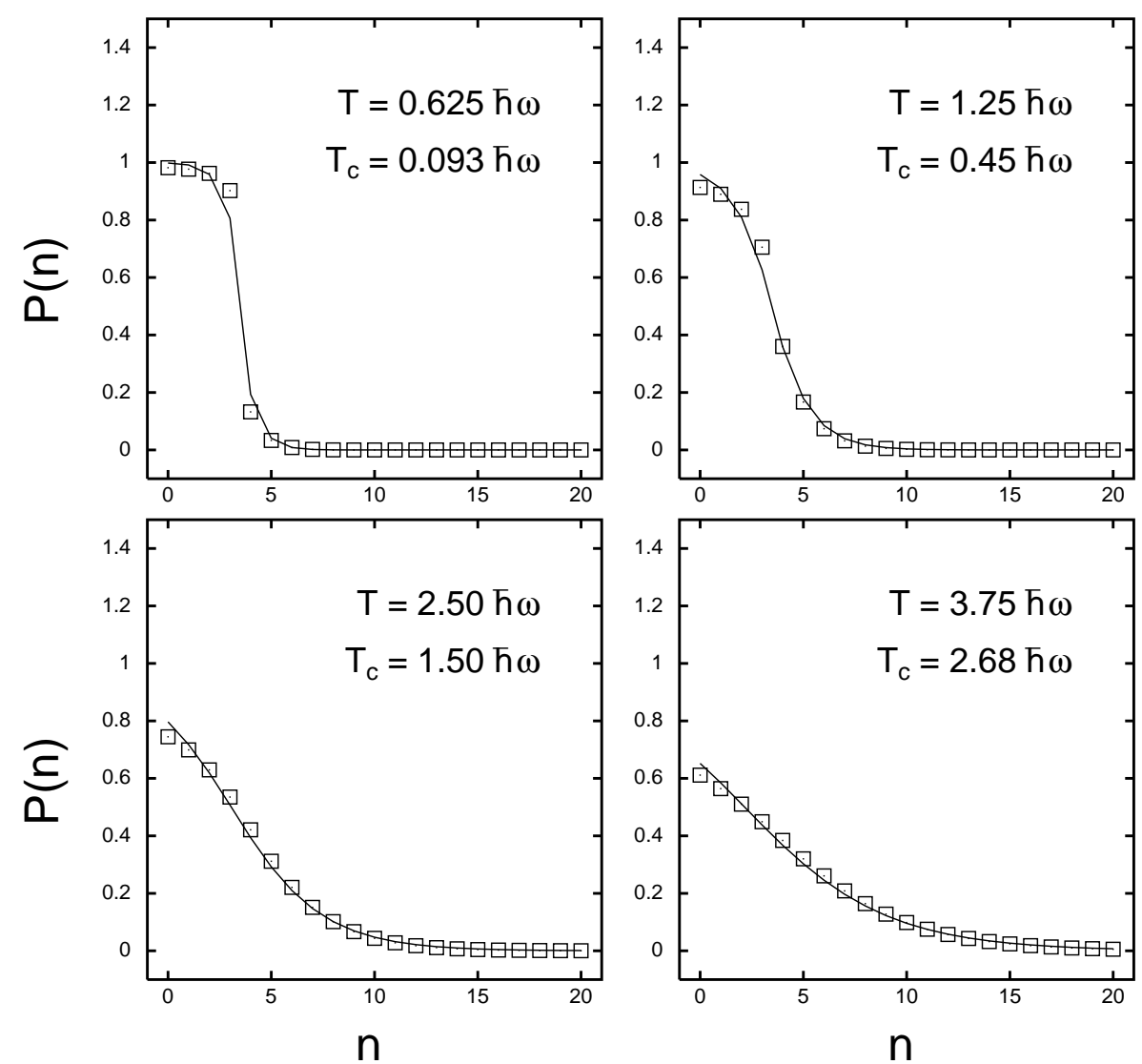

FIG. 2. Occupation probability of the single-particle level $n$ for the one-dimensional harmonic oscillator system with 4 fermions. Squares are the results of $\left\langle\mathcal{P}_{n}(Z)\right\rangle_{T_{c}}$ calculated by assuming the classical canonical statistics of the wave packet centroids. Lines show the quantum mechanical relation $\left\langle\left\langle P_{n}\right\rangle\right\rangle_{T}$. $T$ and $T_{c}$ of each panel are chosen so that they satisfy $\langle\langle H\rangle\rangle_{T}=\langle\mathcal{H}(Z)\rangle_{T_{c}}$. 
The coordinates $Z=\left\{Z_{j}\right\}$ are now canonical coordinates and we have $\operatorname{det}[C]=1$. The calculation of $\langle\mathcal{H}(Z)\rangle_{T_{c}}$ can be executed analytically, giving the result

$$
\begin{aligned}
\mathcal{H}(Z) & =\sum_{j=1}^{A}\left(Z_{j}^{*} Z_{j}+\frac{1}{2}\right) \hbar \omega, \\
\langle\mathcal{H}(Z)\rangle_{T_{c}} & =A\left(T_{c}+\frac{1}{2} \hbar \omega\right) .
\end{aligned}
$$

The calculated result of $\langle\mathcal{H}(Z)\rangle_{T_{c}}$ shows, of course, the classical statistics of the motion of wave packet centroids. The calculation of $\langle\langle H\rangle\rangle_{T}$ for the quantum canonical ensemble can be easily made as shown in ordinary text books of statistical mechanics and we have

$$
\langle\langle H\rangle\rangle_{T}=A\left(\frac{\hbar \omega}{\exp (\hbar \omega / T)-1}+\frac{1}{2} \hbar \omega\right)
$$

From the relation of $\langle\langle H\rangle\rangle_{T}=\langle\mathcal{H}(Z)\rangle_{T_{c}}$, we get the relation between $T$ and $T_{c}$ as follows

$$
T_{c}=\frac{\hbar \omega}{\exp (\hbar \omega / T)-1}
$$

The quantities $\left\langle\mathcal{P}_{n}(Z)\right\rangle_{T_{c}}$ and $\left\langle\left\langle P_{n}\right\rangle\right\rangle_{T}$ can be also easily calculated analytically, and we have

$$
\begin{aligned}
& \mathcal{P}_{n}(Z)=\sum_{j=1}^{A} \frac{\left(Z_{j}^{*} Z_{j}\right)^{n}}{n !} \exp \left(-Z_{j}^{*} Z_{j}\right), \\
& \left\langle\mathcal{P}_{n}(Z)\right\rangle_{T_{c}}=A \frac{\hbar \omega / T_{c}}{\left(1+\hbar \omega / T_{c}\right)^{n+1}}, \\
& \left\langle\left\langle P_{n}\right\rangle\right\rangle_{T}=A\{1-\exp (-\hbar \omega / T)\} \exp (-n \hbar \omega / T) .
\end{aligned}
$$

In getting the results for $\langle\mathcal{H}(Z)\rangle_{T_{c}}$ and $\left\langle\mathcal{P}_{n}(Z)\right\rangle_{T_{c}}$, we have used the formula

$$
\frac{\int d D_{j} d K_{j} \frac{\left(Z_{j}^{*} Z_{j}\right)^{n}}{n !} e^{-\alpha Z_{j}^{*} Z_{j}} e^{-\beta Z_{j}^{*} Z_{j}}}{\int d D_{j} d K_{j} e^{-\beta Z_{j}^{*} Z_{j}}}=\frac{\beta}{(\alpha+\beta)^{n+1}} .
$$

When we use the relation between $T$ and $T_{c}$ of Eq. (32), we can easily prove the equality

$$
\left\langle\mathcal{P}_{n}(Z)\right\rangle_{T_{c}}=\left\langle\left\langle P_{n}\right\rangle\right\rangle_{T} \propto \exp (-n \hbar \omega / T)
$$

Thus we have proved the quantum statistics of the motion of wave packets completely analytically in the case of distinguishable particles under the harmonic oscillator mean field. This proof in the case of distinguishable particles clearly shows that the transition from the classical statistics of wave packet centroids to the quantum statistics of wave packets is due to effects of the wave packet spread, because there is no complexity due to the antisymmetrization. 


\section{AMD STATISTICS FOR THE NUCLEON-EMISSION PROCESS AND INCORPORATION OF WAVE PACKET FLUCTUATION INTO AMD}

In previous sections we have explained a very unique feature of AMD that in the AMD framework two opposite statistics are coexistent: One is the classical statistics which the centroids of nucleon wave packets obey and the other is the quantum statistics which nucleon wave packets obey in their motion described by the AMD wave function. We have pointed out that a key point to understand this coexistent feature of different statistics is to recognize that the temperature of the classical statistics of wave packet centroids is different from the temperature of the quantum statistics of wave packets.

In AMD, the time development of the system is described only through the time development of wave packet centroids which is calculated by the AMD equation of motion and the stochastic two-nucleon collision. The effects of the wave packet spread are taken into account through the use of the AMD wave function for calculating observables. This ordinary AMD framework is expected to work well for the treatment of the internal motion of the nucleus which is free from the nucleon emission. It is because, in the case of the model system under the harmonic oscillator mean field where we have no particle emission, the AMD wave function is just the exact solution of the time-dependent Schrödinger equation. When the ordinary AMD framework is accepted to work well for non-nucleon-emission processes, the AMD statistics should be regarded as being not the classical statistics of wave packet centroids but the quantum statistics of wave packets, because observables are calculated by the use of the AMD wave function which contains effects of the wave packet spread. The statistical ensemble of AMD wave functions is expected to describe well quantum statistical distributions of single-particle level (and/or momentum) occupation probability.

However when we look at the description of the nucleon-emission process by AMD, we find that the description only through the time development of wave packet centroids is not always appropriate. In AMD, a nucleon can be emitted only when the wave packet centroid can go out of the residual nucleus. In quantum mechanics, however, even if the wave packet centroid can not go out of the nucleus, the high momentum component of the wave packet is allowed to go out of the nucleus. Namely, although the wave packet spread should take part explicitly in the dynamics of the nucleon emission, the ordinary AMD framework does not take account of this role of the wave packet spread. It should be noted that the wave packet spread in AMD is large because the energy spread $\left(3 \hbar^{2} \nu / 2 M\right)$ due to the wave packet spread is about $10 \mathrm{MeV}$. The neglect of the nucleon emission due to the high momentum component of the wave packet spread will result, for example, in the small cross section of emitted nucleons or the slowdown of nucleon emission process in the dynamical stage of the reaction. Of course for a high energy nucleon whose wave packet centroid can easily escape from the nucleus, the ordinary AMD description has no serious problem.

According to the above discussion, the emitted nucleons do not reflect the correct momentum distribution inside the nucleus. They reflect only the momentum distribution of wave packet centroids. It means that the statistical properties of the nucleon-emission process can not be quantum mechanical.

In order to check the statistical properties of the nucleon-emission process, we investigated in Ref. [6] the equilibrated coexistent situation of gas and liquid nucleons. For the sake of self-contained discussion, we here recapitulate the method of the investigation in 
Ref. [6]. We put $A_{\text {tot }}$ nucleons into the potential wall with a large radius and give them the total energy $E_{t o t}$. The confinement of nucleons inside the potential wall is made by adding to the AMD Hamiltonian of Eq. (3) the following term

$$
\frac{k}{2} \sum_{j=1}^{A_{t o t}} f\left(\left|\mathbf{D}_{j}-\mathbf{D}_{C M}\right|\right)
$$

with

$$
\begin{aligned}
& f(x)=(x-a)^{2} \theta(x-a), \\
& \mathbf{D}_{j}=\operatorname{Re} \mathbf{Z}_{j} / \sqrt{\nu}, \quad \mathbf{D}_{C M}=\frac{1}{A_{t o t}} \sum_{j=1}^{A_{t o t}} \mathbf{D}_{j}, \\
& a=12 \mathrm{fm}, \quad k=5 \mathrm{MeV} / \mathrm{fm}^{2} .
\end{aligned}
$$

Here $\theta(x)$ is the step function. When we calculate the time evolution of the system for a long time $\left(\sim 10^{5} \mathrm{fm} / \mathrm{c}\right)$, the system is in a phase equilibrium of liquid nucleons and gas nucleons. By liquid nucleons we imply bound nucleons forming a nucleus. The mass number $A_{l i q}$ of liquid nucleons changes with time and we select the moments at which $A_{l i q}$ takes a given value in order to get the statistical properties of the nucleus with the given mass number. We calculate the temperature $T_{g}$ and the long-time average value of the energy $E_{l i q}$ of the liquid nucleons. The temperature $T_{g}$, which should be common to both phases, is calculated as the long-time average value of $\tau$, where $(3 / 2) \tau$ is the kinetic energy (plus the potential energy from the wall) per nucleon in the gas phase. Since the gas phase is dilute, the effect of the Pauli principle is neglected. When there exist some fragments in the gas phase, they are excluded in the calculation of the temperature. In the calculation of the temperature $T_{g}$, i.e. the kinetic energy of gas nucleons, we only use the central value of the wave packet $\operatorname{Im} \mathbf{Z}_{j}$ in the momentum space. It means that we treat gas nucleons as point particles. The treatment of gas nucleons as point particles is consistent with the subtraction of the zeropoint energies of nucleons in the AMD Hamiltonian $\mathcal{H}$ of Eq. (3). It is to be noted that for dilute gas in a large box there is no difference between quantum and classical statistics. The single-particle energy spacing in the box with the size $2 a$ is $\left(\hbar^{2} / 2 M\right)(\pi / 2 a)^{2} \approx 0.35 \mathrm{MeV}$ which is sufficiently small in our present problem.

In Ref. [6] we showed that the relation between $E_{l i q}$ and $T_{g}$ is very close to the linear curve $E_{l i q}^{*} / A_{l i q}=3 T_{g}$ with $E_{l i q}^{*} \equiv E_{l i q}-E_{l i q}$ (ground state). It is displayed again in Fig. 3 . On the other hand, according to the proof in Sec. III, the caloric curve between $E_{l i q}$ and the temperature $T_{c}$ of wave packet centroids of liquid nucleons should be very close to the linear curve $E_{l i q}^{*} / A_{l i q}=3 T_{c}$. Thus we obtain the relation of $T_{g}=T_{c}$. This relation verifies the correctness of our conjecture that in the ordinary AMD the emitted nucleons reflect not the correct momentum distribution of nucleons but only the momentum distribution of wave packet centroids inside the nucleus.

By using the calculated linear relation between $T_{g}$ and $E_{l i q}$ we can discuss conversely as follows. We first regard the gas nucleons as being a thermometer which measures the temperature $T_{c}$ of wave packet centroids of liquid nucleons. Namely we regard that the temperature $T_{g}$ is just the temperature $T_{c}$ of wave packet centroids of liquid nucleons. It is justified because the interaction between the thermometer and the liquid nucleons is 


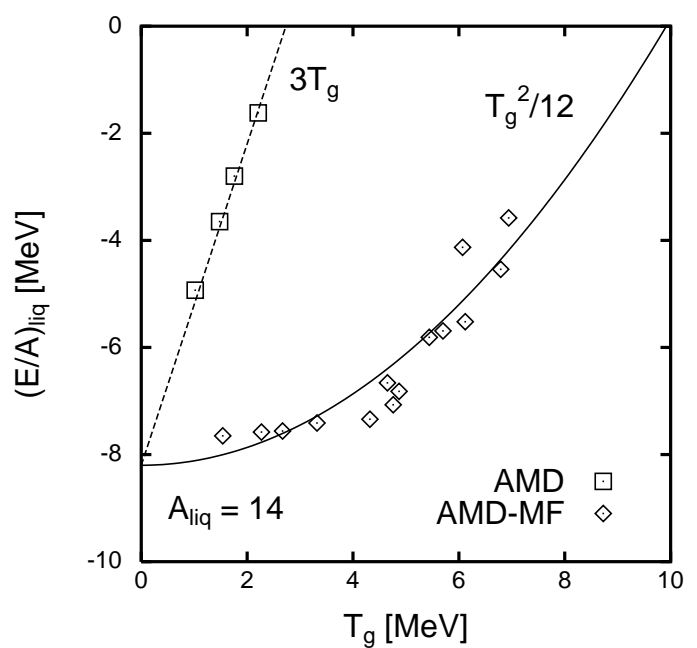

FIG. 3. The statistical property of the excited nucleus calculated in the phase equilibrium of liquid phase and gas phase. Results are shown for the usual AMD (squares) and for AMD-MF (diamonds). Lines of $\left(E^{*} / A\right)_{l i q}=3 T_{g}$ and $T_{g}^{2} /(12 \mathrm{MeV})$ are drawn for the comparison.

described only by the equation of motion of wave packet centroids. Then, the calculated linear relation between $T_{g}$ and $E_{l i q}$ gives us a novel proof of the classical statistics of wave packet centroids. This proof is independent of the proof given in Sec. III by the use of the microcanonical ensemble technique.

Now we discuss the improvement of the description of the nucleon-emission dynamics so that the high momentum component of the wave packet can go out of the nucleus under the situation where the wave packet centroid can not go out of the nucleus. For the improvement, we have to treat the splitting of the wave packet into high and low momentum parts, one going out of the nucleus and the other remaining inside the nucleus. It requires us an introduction of a new stochastic process into AMD. There can be various kinds of new description of the nucleon emission. From the arguments given above, the essential point which any kind of new description should have is that the nucleon emission through the splitting of a wave packet is made according at least to the momentum distribution of the wave packet. In Ref. [6] we presented a new description named AMD-MF in which we apply the new description only when a nucleon is near the nuclear surface and is going to be emitted from the nucleus. For more detailed explanation of the AMD-MF the reader is referred to Ref. [6].

When we introduce a new description of the nucleon emission, we have to check whether the new description ensures correctly the quantum statistics or not for the nucleon-emission process. We discuss the check of the statistics of the AMD-MF also by investigating the equilibrated coexistent situation of gas and liquid nucleons. This investigation was made in Ref. [6] entirely in the same way as discussed above for the check in the case of the usual AMD. The calculated relation between $T_{g}$ and $E_{l i q}$ by the use of AMD-MF was found to be approximated by the caloric curve $E_{l i q}^{*} / A_{l i q}=T_{g}^{2} /(12 \mathrm{MeV})$ with $E_{l i q}^{*} \equiv E_{l i q}-E_{l i q}$ (ground state) which is similar to the empirical Fermi gas caloric curve. It is displayed again in Fig. 3. This result strongly supports that the temperature $T_{g}$ can be regarded as being equal to the temperature $T$ of wave packets of liquid nucleons. We can say that the new description of the nucleon emission in AMD-MF ensures proper quantum statistics for the nucleon-emission 
process.

Here also we can discuss conversely by using the calculated result of $E_{l i q}^{*} / A_{l i q} \approx$ $T_{g}^{2} /(12 \mathrm{MeV})$. We first regard the gas nucleons as being a thermometer which measures the temperature $T$ of wave packets of liquid nucleons. Namely we regard that the temperature $T_{g}$ is just the temperature $T$ of wave packets of liquid nucleons. The justification of this identification is due to the proper treatment of the nucleon emission in AMD-MF. In AMDMF the nucleon emission is made properly according to the momentum distribution of wave packet. Thus we have a good reason to believe that the energy distribution of the emitted nucleons reflects adequately the energy distribution of liquid nucleons which is composed of the convolution of the momentum spread of wave packet and the momentum distribution of wave packet centroids. Then, the calculated caloric curve $E_{l i q}^{*} / A_{l i q} \approx T_{g}^{2} /(12 \mathrm{MeV})$ gives us a novel proof of the quantum statistics of wave packets of liquid nucleons in AMD. This proof is not only independent of the proof given in Sec. IV but also more convincing than it, because the system treated here is a realistic nuclear system while the systems treated in Sec. IV are simple model systems under one-dimensional harmonic oscillator mean field. It is to be stressed here that the present proof elucidates most straightforwardly the fact that the wave packet spread causes the transition from the classical statistics of wave packet centroids to the quantum statistics of wave packets.

Before closing this section, we comment on the treatment of emitted nucleons as point particles in the case of the usual AMD. As mentioned already, this treatment is due to our AMD Hamiltonian $\mathcal{H}$ of Eq. (3) where the zero-point energies of nucleons are subtracted. The subtraction of the zero-point energy means that the zero-point energy is converted into the translational kinetic energy $K^{2} / 2 M$,i.e., that the wave packet in momentum space is forced to shrink into a plane wave or a point particle. If we do not subtract the zero-point energy $3 \hbar^{2} \nu / 2 M$, we can not have low-energy nucleons whose kinetic energy are smaller than $3 \hbar^{2} \nu / 2 M$. It means that the temperature $T_{g}$ of gas nucleons can not be lower than $\hbar^{2} \nu / M$. Moreover, it means that a nucleon can not be emitted from the nucleus if the available energy of a nucleon is smaller than $3 \hbar^{2} \nu / 2 M$. Even when a nucleon can avoid this spurious hindrance of nucleon emission, the emission rate is forced to be spuriously reduced. Now let us consider the usual AMD with the subtraction of zero-point energies of emitted nucleons. Although the subtraction of the zero-point energy favors the nucleon emission compared to the case without subtraction, it still underestimates the nucleon emission rate. The reason is given just by what we discussed in this section. Namely, in the usual AMD the nucleon emission due to the high momentum tail of the nucleon wave packet is neglected. Here, in relation with these discussions, we consider the treatment of the width parameter $\nu$ of the wave packet as dynamical variables as in FMD. In such treatment, the result will become somewhat between two cases with and without the zero-point energy subtraction. Therefore the satisfactory treatment of the nucleon emission will be impossible as long as we require the whole wave packet should go out in the process. Finally we comment on the ad hoc prescription to put the wave packet spread to the emitted nucleons in the usual AMD with the zero-point energy subtraction. This prescription may be regarded as simulating the effect of the emission due to the high momentum tail of the wave packet. It surely improves the fitting of the calculated momentum distribution of emitted nucleons to data in the high momentum region. However this prescription has nothing to do with the emission dynamics and for example the slow emission rate remains untouched. If we calculate the 
gas temperature $T_{g}$ with this ad hoc prescription, the change of $T_{g}$ is simply an increase by $\hbar^{2} \nu / M$ and we have no lower temperature than $\hbar^{2} \nu / M$, showing inadequacy of this temperature.

\section{SUMMARIZING DISCUSSIONS}

We discussed in detail the statistical properties of AMD in general situations including both processes without and with nucleon emissions. The most important point of our discussion was to clarify whether the AMD statistics is quantum mechanical or classical. The basic background for this point of discussion was as follows: AMD is a kind of wave packet molecular dynamics which has been developed in various forms especially in nuclear physics in order to cope with quantum mechanical features of the nuclear system. Since the point particle molecular dynamics used widely in other fields like molecular physics and solid state physics is of classical nature, one tends to think that the wave packet molecular dynamics is also of classical nature. Our basic motivation was to show in a clear way that this understanding is wrong. We stressed that the strongest suggestion that the wave packet molecular dynamics cannot be simply classical comes from the following fact. Namely, the exact solution of the time-dependent Schrödinger equation of the many-body harmonic oscillator system is given by the wave function of the Gaussian wave packet molecular dynamics. This fact is well known for the system of distinguishable particles. This fact is, however, also true for the system of fermions as can be easily proved.

In order to discuss the statistical properties of wave packet molecular dynamics, we have to clarify the roles of two ingredients of wave packet molecular dynamics, i.e. wave packet centroids and wave packet spread. Needless to say, one cannot at all make light of the role of wave packet spread in the discussion of statistical properties of wave packet molecular dynamics. For example, the momentum distribution of the system can not be described without wave packet spread. In general, in wave packet molecular dynamics physical quantities are not described simply by using only wave packet centroids but by using the wave function including the wave packet spread.

One of the basic points of this paper was to elucidate the fact that the presence of two ingredients gives rise to a very characteristic feature of the AMD statistics. It is the coexistence of mutually opposite two statistics in the AMD framework, one being the classical statistics which wave packet centroids obey in their motion described by the AMD equation of motion and the other being the quantum statistics which wave packets obey in their motion described by AMD wave function. The coexistence of the opposite two statistics is also true for the wave packet molecular dynamics of distinguishable particles and is a very important and novel character of the wave packet molecular dynamics which constitutes a decisive difference from the point particle molecular dynamics.

The fact that the statistics of wave packet centroids in AMD is classical in spite of the antisymmetrization was proved in two independent ways in this paper. Both of the proofs were different from the proof of Ref. [3] by Ohnishi and Randrup and were made in the case of realistic many-nucleon systems interacting with effective two-nucleon force. One was a rather direct proof by the use of the microcanonical ensemble technique. The other was a proof by the use of a thermometer made of gas nucleons which measures the temperature of 
the motion of wave packet centroids inside the nucleus. The reason why the thermometer measures the temperature of the wave packet centroids is because the interaction between the thermometer and the nucleons inside the nucleus is governed only by the motion of wave packet centroids without any effects of wave packet spread.

The fact that the AMD statistics of wave packets including effects of wave packet spread is quantum mechanical was also given two independent proofs in this paper. Both of them are different from the proof of Ref. [4] by Schnack and Feldmeier. One was a proof in the case of one-dimensional harmonic oscillator systems of fermions and also of distinguishable particles. We calculated the occupation probability of the harmonic oscillator single-particle level on the basis of the fact that wave packet centroids obey classical statistics. We then showed that the calculated values of the occupation probability were almost the same as the values given by the quantum canonical ensemble average. What was decisively important in this proof was the recognition of the fact that the temperature of the classical statistics of wave packet centroids is different from the temperature which characterizes the quantum statistics of wave packets. A merit of this proof is that it clarifies the relation between the classical statistics of wave packet centroids and the quantum statistics of wave packets, or in other words, it clarifies how the quantum statistics of wave packets emerges from the classical statistics of wave packet centroids. The other proof was by the use of a new thermometer made of gas nucleons which measures the temperature of the motion of wave packets inside the nucleus. The new thermometer interacts with the nucleus now through a new mechanism of the nucleon emission from the nucleus. The nucleon emission from the nucleus is made not by the ordinary AMD but by the modified AMD named AMD-MF which was proposed in Ref. [6]. In the AMD-MF, the nucleon emission is governed not by the motion of the wave packet centroids but by the momentum distribution due to the wave packet spread. A merit of this proof is that it clarifies most straightforwardly the role of the wave packet spread for the transition from the classical statistics of wave packet centroids to the quantum statistics of wave packets.

The temperature which is relevant to the calculated observables in AMD is of course not the temperature $T_{c}$ of wave packet centroids but the temperature $T$ of wave packets. The serious mistake of the work of Ref. [3] by Ohnishi and Randrup is that they regarded the temperature $T_{c}$ of wave packet centroids as being the temperature relevant to the calculated observables. Based on this mistake they insisted a wrong opinion that one should correct the AMD framework even for the harmonic oscillator many-body system for which the AMD wave function gives the exact solution of the time-dependent Schrödinger equation.

The conclusion of this paper about the character of the AMD statistics can be said as follows: As far as the effects of the wave packet spread are duely taken into account, the AMD statistics is quantum mechanical. For processes without nucleon emission, namely for the motion confined inside the nucleus, the ordinary AMD takes due account of the wave packet spread and the AMD statistics is quantum mechanical without any need of essential modification. However, for the nucleon-emission process, the ordinary AMD does not duely take account of the wave packet spread. Namely in the ordinary AMD, the emitted nucleons reflect not the momentum distribution of nucleons inside the nucleus but that of wave packet centroids inside the nucleus. Hence the statistics of the ordinary AMD for the nucleon-emission process is classical. In order to recover the quantum statistics also for the nucleon-emission process, the description of the nucleon-emission process of the ordinary 
AMD should be modified so that the nucleon emission is made according to the momentum distribution of nucleons inside the nucleus which is the convolution of the momentum spread of the wave packet and the momentum distribution of wave packet centroids. The AMD-MF method proposed in Ref. [6] is one of such modified versions of AMD and it was verified that that the statistics of the AMD-MF for the nucleon-emission process is actually quantum mechanical.

What is to be noticed in the above discussion is that the reason of the classical statistics of the usual AMD for the nucleon-emission process is originating not from any complex mechanism but from a simple mechanism of the single-particle motion of emission. Thus we have been able to recover the proper quantum statistics for the nucleon-emission process by a modification of the single-particle dynamics. In the modification, a proper incorporation of the wave packet spread into the dynamical process should be made. Here, however, this modification should not be confused with the treatment of the width parameters of wave packets as time-dependent dynamical variables.

The modification of the AMD by the AMD-MF can be generalized so that we can improve the description of the cluster emission process and furthermore that of the nucleon-transfer process between the projectile and the target in addition to that of the nucleon-emission process. We have already formulated such extension and have found that fragmentation processes are largely influenced by the extended modification. We will discuss these in other papers. 


\section{REFERENCES}

[1] A. Ono, H. Horiuchi, Toshiki Maruyama and A. Ohnishi, Phys. Rev. Lett. 682898 (1992); Prog. Theor. Phys. 87, 1185 (1992); Phys. Rev. C47 2652 (1993).

[2] A. Ono, H. Horiuchi, and Toshiki Maruyama, Phys. Rev. C48 2946 (1993); A. Ono and H. Horiuchi, Phys. Rev. C51, 299 (1995); E. I. Tanaka, A. Ono, H. Horiuchi, Tomoyuki Maruyama, and A. Engel, Phys. Rev. C52, 316 (1995); A. Engel, E. I. Tanaka, Tomoyuki Maruyama, A. Ono and H. Horiuchi, Phys. Rev. C (1995) to be published.

[3] A. Ohnishi and J. Randrup, Nucl. Phys. A565, 474 (1993).

[4] J. Schnack and H. Feldmeier, preprint GSI-95-34 (1995).

[5] H. Feldmeier, Nucl. Phys. A515, 147 (1990); H. Feldmeier, K. Bieler, and J. Schnack, Nucl. Phys. A586, 493 (1995); H. Feldmeier and J. Schnack, Nucl. Phys. A583, 347 (1995).

[6] A. Ono and H. Horiuchi, preprint RIKEN-AF-NP-202 (1995).

[7] W. Pauli, Pauli Lectures on Physics, Vol.4, Statistical Mechanics, edited by C. P. Enz, translated by H. R. Lewis and S. Margulies, (MIT Press, Cambridge, Mass., 1973).

[8] A. Volkov, Nucl. Phys. 75, 33 (1965). 